Arq. Bras. Med. Vet. Zootec., v.65, n.2, p.490-496, 2013

\title{
Comportamento ingestivo de cordeiros alimentados com torta de macaúba
}

\author{
[Ingestive behavior of lambs fed with macauba meal] \\ R.A. Azevedo ${ }^{1}$, L.M.A. Rufino ${ }^{2}$, A.C.R. Santos ${ }^{1}$, C.S. Ribeiro Júnior ${ }^{3}$, \\ N.M. Rodriguez ${ }^{1}$, L.C. Geraseev ${ }^{1, *}$ \\ ${ }^{1}$ Instituto de Ciências Agrárias - Universidade Federal de Minas Gerais - Montes Claros, MG \\ ${ }^{2}$ Universidade Federal de Viçosa - Viçosa, MG \\ ${ }^{3}$ Universidade Estadual Paulista - Jaboticabal, SP
}

\begin{abstract}
RESUMO
Avaliaram-se os efeitos de diferentes porcentagens (0, 10, 20 e 30\%) de inclusão da torta de macaúba (TM) no comportamento ingestivo de 24 cordeiros Santa Inês, com média de cinco meses de idade e peso vivo de $23,9 \mathrm{~kg}$, distribuídos em delineamento em blocos ao acaso, com quatro tratamentos e seis repetições, durante 60 dias de confinamento. Foram avaliados os tempos médios despendidos com alimentação, ruminação (TR), ócio (TO), tempo de mastigação total (TMT), eficiência de alimentação $\left(\mathrm{EA}_{\mathrm{MS}}\right.$ e $\left.\mathrm{EA}_{\mathrm{FDN}}\right)$ e eficiência de ruminação, além do número de bolos ruminados (NBR) e número diário de mastigações merícicas (MMnd). A adição de $\mathrm{TM}$ à dieta aumentou linearmente o TR, TMT, NBR, MMnd e $\mathrm{EA}_{\mathrm{FDN}}$ e reduziu de forma linear o TO, em consequência do maior teor de fibra em detergente neutro das dietas com o coproduto. Mesmo com o aumento no TMT, não foi verificada diferença no consumo e na eficiência de ruminação e alimentação da matéria seca, possivelmente em razão da baixa efetividade de fibra da TM, demonstrando o potencial de utilização desse coproduto na dieta de ovinos em crescimento.
\end{abstract}

Palavras-chave: confinamento, coprodutos, terminação, ovinos

\begin{abstract}
The effects of different percentages (0, 10, 20 and 30\%) of inclusion of macauba meal (MM) on the ingestive behavior of 24 Santa Inês male lambs, averaging five months of age and $23.9 \mathrm{~kg}$ of initial body weight were studied. The animals were allotted to a completely randomized block design with four treatments and six replications during 60 days of confinement. The variables measured were time spent in rumination (TR), idle time (IT), total chewing (TC), power efficiency $\left(P E_{D M}\right.$ and $\left.P E_{N D F}\right)$ and efficiency of rumination, as well as the number of ruminated bolus $(N R B)$ and daily number of chews $(C n d)$. The addition of MM to the diet increased $T R, T C, N R B, C n b$ and $P E_{N D F}$ linearly, and reduced IT linearly, as a result of higher content of neutral detergent fiber in the diet. Although there was an increase in TC, no difference was verified in the consumption and efficiency of rumination and feeding of DM, possibly due to the low efficiency of fiber, which demonstrates the potential use of this coproduct in the diet of growing sheep.
\end{abstract}

Keywords: coproduct, feedlot, termination, sheep

\section{INTRODUÇÃO}

Coprodutos são alimentos alternativos para a alimentação de ruminantes, os quais apresentam capacidade de utilizar resíduos vegetais para suprir seus requisitos nutricionais, além de substituírem, de forma parcial ou total, os alimentos concentrados padrão das dietas, como o milho e o farelo de soja (Pires et al., 2005; Silva et al., 2005). Segundo Mendonça et al. (2004), por apresentar composição química e física diferente dos alimentos concentrados padrão, a utilização de coprodutos deve ser acompanhada por avaliações do comportamento ingestivo dos animais, pois estas servem como ferramenta para avaliação de dietas,

Recebido em 14 de agosto de 2011

Aceito em 10 de agosto de 2012

*Autor para correspondência (corresponding author)

E-mail: lgeraseev@gmail.com 
possibilitando ajustar o manejo alimentar para obtenção de melhores desempenhos.

A torta da macaúba (Acrocomia aculeata (Jacq.) Lodd. Ex Mart.), resíduo da extração do óleo da polpa do fruto, pode ser um coproduto viável para a alimentação de ovinos, e análises químicas preliminares realizadas nas tortas residuais do coco da macaúba demonstram que estas poderiam ser aproveitadas na alimentação de ruminantes (Azevedo et al. 2012). Entretanto, não há trabalhos na literatura consultada sobre a utilização desse resíduo na alimentação de ovinos, sendo necessários estudos para avaliar os efeitos da sua utilização sobre o comportamento ingestivo dos animais.

Ao avaliarem o comportamento ingestivo de ovinos Santa Inês alimentados com diferentes teores de farelo de cacau, Carvalho et al. (2008) verificaram que a eficiência de alimentação ( $\mathrm{g}$ MS e FDN/hora) não foi influenciada pela inclusão do coproduto. Entretanto, houve alterações na eficiência de ruminação (g MS e FDN/bolo). Fontenelle et al. (2011) constataram que cordeiros da raça Santa Inês alimentados com diferentes níveis de energia metabolizável apresentaram redução linear nos tempos de alimentação e mastigação total em função do aumento dos níveis energéticos das rações; já o número de bolos ruminais, mastigações merícicas e tempo de mastigações merícicas por bolo ruminal não foram influenciados.

Objetivou-se com este trabalho avaliar o comportamento ingestivo de ovinos da raça Santa Inês, em confinamento, alimentados com dietas compostas por diferentes porcentagens de inclusão da torta de macaúba.

\section{MATERIAL E MÉTODOS}

Foram utilizados 24 cordeiros da raça Santa Inês, machos, não castrados, com idade média de cinco meses e peso vivo médio inicial de $23,9 \pm 0,6 \mathrm{~kg}$. Inicialmente os animais foram vermifugados (ivermectina) e vacinados contra clostridiose após a identificação e a pesagem. Os animais foram distribuídos em blocos ao acaso, com quatro tratamentos e seis repetições. Utilizou-se o peso corporal inicial dos animais para definição dos blocos. O experimento teve duração de 70 dias, sendo os 10 primeiros dias destinados à adaptação dos animais ao ambiente e à dieta, e os 60 dias restantes para coletas dos dados. Os animais foram confinados individualmente em baias de $1,2 \mathrm{~m}^{2}$, providas com comedouros e bebedouros.

Forneceu-se a alimentação duas vezes ao dia, às $07 \mathrm{~h}$ e às $17 \mathrm{~h}$, ajustada de forma a manter as sobras em $20 \%$ do oferecido. As dietas experimentais foram formuladas de acordo com as recomendações do NRC (National..., 2007), com porcentagens crescentes de torta de macaúba - 0; 10; 20 e $30 \% \quad-$, sendo isonitrogenadas, com $15 \%$ de PB com base na MS. As dietas foram constituídas de silagem de sorgo na proporção de $30 \%$ e $70 \%$ de concentrado à base de milho, farelo de soja, caroço de algodão, torta de macaúba, calcário calcítico, fosfato bicálcico e premix mineral e vitamínico (Tab. 1 e 2). A torta de macaúba (TM) utilizada foi doada pela Cooperativa de Pequenos Produtores Rurais de Riacho D'Antas e Adjacências, localizada em Montes Claros, MG. $\mathrm{Na}$ Tabela 1 está apresentada a composição percentual dos ingredientes nas dietas experimentais e, na Tabela 2, a composição bromatológica das dietas experimentais e da TM.

Tabela 1. Composição percentual dos ingredientes nas dietas experimentais, com base na matéria seca

\begin{tabular}{lcccc}
\hline \multicolumn{1}{c}{ Ingrediente } & \multicolumn{4}{c}{ Inclusão de torta de macaúba (\% da MS) } \\
& $0 \%$ & $10 \%$ & $20 \%$ & $30 \%$ \\
\hline Silagem de sorgo & 30,00 & 30,00 & 30,00 & 30,00 \\
Farelo de soja & 14,98 & 15,30 & 15,61 & 15,93 \\
Milho & 48,65 & 38,40 & 28,14 & 17,89 \\
Caroço de algodão & 5,00 & 5,00 & 5,00 & 5,00 \\
Torta de macaúba & 0,00 & 10,00 & 20,00 & 30,00 \\
Fosfato bicálcico & 0,33 & 0,33 & 0,34 & 0,34 \\
Calcário & 0,74 & 0,67 & 0,61 & 0,54 \\
Sal comum & 0,28 & 0,28 & 0,28 & 0,28 \\
Premix & 0,02 & 0,02 & 0,02 & 0,02 \\
\hline
\end{tabular}


Tabela 2. Composição bromatológica das dietas experimentais e da torta de macaúba

\begin{tabular}{lccccc}
\multirow{2}{*}{\multicolumn{1}{c}{ Item }} & \multicolumn{3}{c}{ Inclusão de torta de macaúba (\% da MS) } & \multirow{2}{*}{ TM } \\
\cline { 2 - 5 } & $0 \%$ & $10 \%$ & $20 \%$ & $30 \%$ & \\
\hline Matéria seca (\% da matéria natural) & 72,92 & 73,94 & 74,30 & 74,80 & 94,96 \\
Matéria orgânica (\% da MS) & 95,72 & 95,32 & 94,81 & 94,14 & 96,55 \\
Proteína bruta (\% da MS) & 15,01 & 15,88 & 15,81 & 15,74 & 8,18 \\
Nutrientes digestíveis totais ${ }^{1}$ (\% da MS) & 66,34 & 65,37 & 64,59 & 63,98 & 68,10 \\
Extrato etéreo (\% da MS) & 3,90 & 4,95 & 6,20 & 6,79 & 14,95 \\
Fibra em detergente neutro (\% da MS) & 32,09 & 36,35 & 40,88 & 43,17 & 62,03 \\
Fibra em detergente ácido (\% da MS) & 16,75 & 20,33 & 24,96 & 27,23 & 49,39 \\
Carboidratos totais (\% da MS) & 76,81 & 74,49 & 72,80 & 71,61 & 73,42 \\
Carboidratos não fibrosos (\% da MS) & 44,72 & 38,14 & 31,92 & 28,44 & 11,39 \\
\hline
\end{tabular}

${ }^{1}$ Nutrientes digestíveis totais (NDT = 40,2625 + 0,1969PB + 0,4028 CNF + 1,903EE - 0,1379 FDA; Weiss, 1998).

A ingestão de alimentos foi calculada pela diferença diária dos pesos da dieta oferecida e das sobras. Amostras dos alimentos fornecidos e das respectivas sobras das dietas foram coletadas diariamente, sendo identificadas e acondicionadas em freezer para posteriores análises laboratoriais. Todas as amostras foram pré-secadas em estufa ventilada a $55^{\circ} \mathrm{C}$ e, posteriormente, moídas em moinho de facas com peneira de $1 \mathrm{~mm}$, acondicionadas em frascos identificados, para posterior determinação de MS e FDN, seguindo a metodologia descrita por Silva e Queiroz (2002).

Todos os animais foram submetidos a três observações visuais, por quatro observadores treinados e posicionados estrategicamente, de forma a não incomodar os animais, para avaliação do comportamento ingestivo no início, meio e fim do experimento. As observações ocorreram a cada cinco minutos, durante 24 horas, subdivididas em três períodos de oito horas, para determinação do tempo despendido em ingestão, ruminação e ócio, conforme metodologia citada por Johnson e Combs (1991), totalizando 384 observações por período.

No mesmo dia das observações foi realizada a contagem do número de mastigações merícicas por bolo ruminado $\mathrm{MMnb}\left(\mathrm{n}^{\circ} / \mathrm{bolo}\right)$ e do tempo despendido para ruminação de cada bolo MMtb (seg/bolo), com a utilização de um cronômetro digital. Para obtenção das médias das mastigações e do tempo, foram feitas as observações de três bolos ruminais em três períodos diferentes do dia (8-10, 14-16 e 2022h). O galpão foi mantido com iluminação artificial durante todo o período noturno do experimento.

Calculou-se a quantidade de MS e FDN por bolo alimentar pela divisão do consumo médio individual de cada fração pelo número de bolos ruminados por dia (em 24 horas). O número de bolos ruminados foi obtido pela divisão do tempo total de ruminação pelo tempo médio para ruminar cada bolo. A eficiência de alimentação (EA) e ruminação (ER) em g/MS/h e g/FDN/h foi calculada por meio da divisão do consumo médio diário de MS e FDN pelo tempo total despendido em alimentação e/ou ruminação durante 24 horas, respectivamente. Estas e outras variáveis determinadas neste experimento, como número de mastigações merícicas por dia (MMnd) e o tempo de mastigação total (TMT), foram obtidas conforme metodologia descrita por Polli et al. (1996) e Burger et al. (2000).

Os resultados foram submetidos à análise de variância e de regressão utilizando-se o SAEG (Sistema, 2007).

\section{RESULTADOS E DISCUSSÃO}

$\mathrm{O}$ aumento nas porcentagens de TM nas dietas influenciou $(\mathrm{P}<0,05)$ os tempos despendidos com ruminação, ócio e mastigação total (Tab. 3). Entretanto, não ocorreu influência sobre o tempo despendido com alimentação em 24 horas $(\mathrm{P}>0,05)$. 
Tabela 3. Médias e equações de regressão para consumos de matéria seca (CMS) e de fibra em detergente neutro (CFDN) em 24 horas; tempo médio despendido com alimentação (TA), ruminação (TR), ócio (TO), mastigação total (TMT) e eficiências de alimentação (EA $A_{M S}$ e $\left.E_{F D N}\right)$ e ruminação $\left(E_{M S}\right.$ e $\left.E_{F D N}\right)$ em função da porcentagem de inclusão da torta de macaúba na dieta de cordeiros confinados

\begin{tabular}{|c|c|c|c|c|c|c|c|}
\hline \multirow{2}{*}{ Variável } & \multicolumn{4}{|c|}{ Torta de macaúba (\%) } & \multirow{2}{*}{$\mathrm{EPM}^{1}$} & \multicolumn{2}{|c|}{ Efeito } \\
\hline & $0 \%$ & $10 \%$ & $20 \%$ & $30 \%$ & & $\mathrm{~L}$ & $\mathrm{Q}$ \\
\hline CMS (g/dia) & 1093,56 & 1093,30 & 1132,85 & 1179,46 & 31,20 & 0,29 & 0,71 \\
\hline CFDN (g/dia) ${ }^{2}$ & 313,68 & 366,10 & 446,74 & 489,29 & 17,76 & 0,00 & 0,82 \\
\hline $\mathrm{TA}$ (min/dia) & 163,48 & 146,48 & 149,47 & 156,53 & 13,05 & 0,88 & 0,66 \\
\hline $\mathrm{TR}(\mathrm{min} / \mathrm{dia})^{3}$ & 450,25 & 511,95 & 591,66 & 561,27 & 23,77 & 0,04 & 0,31 \\
\hline $\mathrm{TO}(\mathrm{min} / \mathrm{dia})^{4}$ & 826,27 & 781,56 & 698,86 & 722,20 & 23,62 & 0,05 & 0,45 \\
\hline $\mathrm{TMT}(\mathrm{min} / \mathrm{dia})^{5}$ & 613,73 & 658,43 & 741,13 & 717,82 & 23,52 & 0,03 & 0,11 \\
\hline $\mathrm{EA}_{\mathrm{MS}}(\mathrm{gMS} / \mathrm{h})$ & 484,34 & 494,24 & 557,64 & 586,44 & 51,71 & 0,43 & 0,93 \\
\hline $\mathrm{EA}_{\mathrm{FDN}}(\mathrm{gFDN} / \mathrm{h})^{6}$ & 138,42 & 165,14 & 219,29 & 241,98 & 20,36 & 0,04 & 0,95 \\
\hline $\mathrm{ER}_{\mathrm{MS}}(\mathrm{gMS} / \mathrm{h})$ & 161,17 & 129,29 & 115,01 & 133,75 & 8,86 & 0,23 & 0,15 \\
\hline $\mathrm{ER}_{\mathrm{FDN}}(\mathrm{gFDN} / \mathrm{h})$ & 46,25 & 43,31 & 45,32 & 55,37 & 2,76 & 0,24 & 0,24 \\
\hline
\end{tabular}

O efeito linear sobre o tempo gasto na ruminação com a inclusão do coproduto, observado neste estudo, foi consequência da elevação dos teores de fibra em detergente neutro nas dietas com o coproduto. Segundo Church (1988) e Mertens (1997), ao receber dietas com teores mais altos de FDN, os animais necessitam de maior tempo para ruminação em razão da maior necessidade de processar a fibra da dieta e por apresentarem maiores estímulos às atividades mastigatórias.

Frequentemente são observadas alterações nos tempos despendidos nas atividades de alimentação e ruminação em pesquisas nas quais os tratamentos apresentaram dietas com variações nos teores de fibra (Carvalho et al., 2006). Carvalho et al. (2008) observaram semelhança entre os tempos despendidos com alimentação, ruminação e ócio ao se fornecer diferentes porcentagens de farelo de cacau a ovinos da raça Santa Inês. Os autores justificam esse resultado pelo fato das dietas fornecidas serem isonitrogenadas e apresentarem pequenas variações nos teores de fibra, diferentemente do presente estudo, em que o teor de fibra em detergente neutro nas dietas variou de 32,09 a $62,03 \%$ da MS.

Apesar do aumento da porcentagem de FDN das dietas com a inclusão da TM, não foi observado efeito significativo sobre o TA $(\mathrm{P}>0,05)$. Tal resultado difere dos encontrados por Carvalho (2002), que observou aumento linear $(\mathrm{P}<0,01)$ no tempo gasto com alimentação à medida que o teor de fibra foi elevado na dieta de cabras lactantes, e por Gonçalves et al. (2001), que, ao trabalharem com cabras leiteiras alimentadas com diferentes relações volumoso:concentrado, também observaram aumento nos tempos despendidos com alimentação e ruminação, e redução no tempo despendido em ócio com a elevação dos teores de fibra nas dietas. Contudo, Cardoso et al. (2006) e Carvalho et al. (2006) não observaram influência das porcentagens de FDN ( $\mathrm{P}>0,05)$ sobre o tempo despendido pelos animais em alimentação, ruminação, ócio e mastigação total.

O aumento linear no tempo de mastigação total e a redução no tempo despendido em ócio em função da inclusão da TM nas dietas podem ser explicados pelo aumento no tempo gasto com ruminação. Segundo Van Soest (1994), o tempo gasto em ruminação é proporcional ao teor de parede celular dos alimentos; assim, ao elevar-se a porcentagem de FDN das dietas, haverá aumento no tempo despendido com ruminação e redução do tempo despendido em ócio.

Como verificado na Tab. 3, o consumo de matéria seca (CMS, g/dia) foi semelhante $(\mathrm{P}>0,05)$ entre os tratamentos, com valor médio de $1124,79 \mathrm{~g} /$ dia. Da mesma forma, não foi observada $(\mathrm{P}>0,05)$ influência das dietas sobre a eficiência de alimentação da MS. O consumo de MS (g/dia) semelhante entre os tratamentos justifica os resultados obtidos para as eficiências 
de ingestão de MS, que é diretamente relacionada ao consumo expresso em $\mathrm{g} / \mathrm{dia}$.

Mesmo as dietas apresentando teores de FDN e tempos despendidos com ruminação diferentes, a eficiência de ruminação da MS e da FDN não foram influenciadas $(\mathrm{P}>0,05)$. Segundo Dado e Allen (1995), a eficiência digestiva aumenta quando ocorre o processamento da digesta ruminal. Sendo assim, era esperado que a eficiência de ruminação, em $\mathrm{g} M \mathrm{MS} / \mathrm{h}$, decrescesse com o aumento no consumo de FDN pelos animais, pois o aumento da concentração de constituintes da parede celular nas dietas eleva o número de mastigações merícicas por bolo alimentar e, consequentemente, o tempo despendido com a ruminação (Dulphy et al., 1980; Cardoso et al., 2006), reduzindo, assim, o número de ruminações.

A eficiência de ruminação é um comportamento importante no controle da utilização de alimentos fibrosos e pode restringir o uso de alimentos de baixa qualidade, comprometendo a produção animal (Welch, 1982). Segundo Fontenelle et al. (2011), o prolongamento da atividade de ruminação nem sempre compensa a redução na eficiência de ruminação.

Mesmo ocorrendo variação nos teores de FDN das dietas experimentais e no TR com a inclusão da torta de macaúba nas dietas, essas variações não foram suficientes para provocar alterações no CMS e na eficiência de ruminação ( $\mathrm{g}$ de MS e FDN/bolo). Um dos fatores que poderia explicar esse efeito sobre a ruminação é o processamento físico da torta de macaúba. A moagem desse coproduto resulta em partículas com tamanho semelhante ao dos alimentos concentrados padrão, constituídos de milho moído e farelo de soja. Esses resultados demonstram que a utilização desse coproduto possui perfil de fibra fisicamente efetiva interessante, sendo a fração do alimento que estimula a atividade de mastigação pelos animais (Allen, 1997), podendo contribuir na eficiência de alimentação e de ruminação destes.

Mendes et al. (2010), ao avaliarem os efeitos da substituição da FDN do bagaço de cana-deaçúcar in natura pela FDN da casca de soja em dietas com alta proporção de concentrado para cordeiros, encontraram tempos de ruminação diferentes para as dietas com bagaço de cana. No mesmo trabalho verificou-se superioridade das dietas contendo bagaço de cana in natura sobre a atividade de mastigação quando comparadas com as dietas com casca de soja. Isso se justifica pelo fato de a casca de soja ter apresentado menor efetividade de fibra em relação ao bagaço de cana in natura.

O número de mastigações merícicas por bolo ( $\mathrm{n}^{\circ} /$ bolo) e o tempo de mastigação merícica por bolo ruminado (seg/bolo) não sofreram alterações $(\mathrm{P}>0,05)$ com a inclusão da torta de macaúba (Tab. 4). Já o número de bolos ruminados ( $\mathrm{n} \%$ dia) e o número de mastigações merícicas ( $\mathrm{n} /$ dia $)$ aumentaram $\quad(\mathrm{P}<0,05)$ linearmente com a inclusão do coproduto na alimentação dos animais.

Tabela 4. Médias e equações de regressão para o número de bolos ruminados (NBR), número de mastigações merícicas por bolo (MMnb), número de mastigações merícicas por dia (MMnd), tempo de mastigações merícicas por bolo (MMtb), gramas de matéria seca por bolo ruminado (GMSB) e gramas de fibra em detergente neutro por bolo ruminado (GFDNB), em função da porcentagem de inclusão da torta de macaúba na dieta de cordeiros confinados

\begin{tabular}{|c|c|c|c|c|c|c|c|}
\hline \multirow{2}{*}{ Variável } & \multicolumn{4}{|c|}{ Torta de macaúba (\%) } & \multirow{2}{*}{$\mathrm{EPM}^{1}$} & \multicolumn{2}{|c|}{ Efeito } \\
\hline & $0 \%$ & $10 \%$ & $20 \%$ & $30 \%$ & & $\mathrm{~L}$ & Q \\
\hline $\operatorname{NBR}\left(\mathrm{n}^{\circ} / \mathrm{dia}\right)^{2}$ & 566,65 & 761,88 & 795,78 & 781,82 & 36,04 & 0,03 & 0,11 \\
\hline MMnb (nºlbolo) & 65,07 & 62,49 & 57,24 & 62,8287 & 1,49 & 0,68 & 0,59 \\
\hline MMnd (n $\%$ dia $)^{3}$ & 36323,4 & 44420,67 & 50626,03 & 47875,66 & 2211,53 & 0,03 & 0,19 \\
\hline MMtb (seg/bolo) & 47,83 & 40,85 & 44,60 & 43,91 & 1,44 & 0,54 & 0,29 \\
\hline GMSB (g/bolo) & 2,07 & 1,47 & 1,43 & 1,63 & 0,10 & 0,17 & 0,06 \\
\hline GFDNB (g/bolo) & 0,59 & 0,49 & 0,56 & 0,68 & 0,03 & 0,30 & 0,12 \\
\hline
\end{tabular}

${ }^{\mathrm{I}}$ Erro padrão da média; ${ }^{2} \mathrm{y}=6,7943 \mathrm{x}+624,62\left(\mathrm{R}^{2}=66,58\right) ;{ }^{3} \mathrm{y}=408,63 \mathrm{x}+38682\left(\mathrm{R}^{2}=72,35\right)$. 
Os aumentos verificados para NBR e MMnd estão diretamente correlacionados com $\mathrm{o}$ aumento linear verificado para o tempo despendido com ruminação, pois dietas com maiores concentrações de fibra aumentam o estímulo de ruminação (Macedo et al., 2007). Os resultados verificados para o MMnb também podem ser explicados pela baixa efetividade física que esse coproduto apresenta, pois não houve aumento do MMnb, mesmo sendo verificado aumento do CFDN com a inclusão da TM nas dietas.

Segundo Mertens (1997), a atividade de mastigação é uma característica que reflete as propriedades químicas e físicas dos alimentos. Carvalho et al. (2008) verificaram comportamento quadrático para o número de bolos ruminados por dia ( $\mathrm{n} / \mathrm{dia})$ e para o tempo médio gasto em mastigações por bolo ruminado (seg) ao testarem diferentes porcentagens de farelo de cacau nas dietas de ovinos.

Macedo et al. (2007), ao testarem diferentes porcentagens de substituição da silagem de sorgo pelo bagaço de laranja, não observaram influência $(\mathrm{P}>0,05)$ sobre o tempo de mastigação total (min/dia), número de bolos ruminais por dia, número de mastigações merícicas por bolo e tempo médio de mastigações por bolo (seg/bolo), sendo encontrada diferença somente no número de mastigações merícicas por dia (MMnd), com efeito linear decrescente $(\mathrm{P}<0,05)$, justificandose pela redução do teor dietético de FDN, pois, segundo os autores, dietas com menor concentração de fibra diminuem o estímulo de ruminação e aceleram a taxa de passagem dos alimentos pelo trato digestivo.

Os resultados verificados para o MMtb, GMSB e GFDNB estão correlacionados com as observações similares entre as eficiências de ruminação da MS e do FDN, possivelmente devido ao seu tamanho de partícula similar aos concentrados padrão e à sua baixa efetividade de fibra.

\section{CONCLUSÕES}

A inclusão da torta de macaúba em dietas com porcentagens de até $30 \%$ para ovinos da raça Santa Inês influencia o comportamento ingestivo dos animais, alterando principalmente o tempo de ruminação, entretanto, não compromete o consumo e o desempenho dos animais. Os resultados demonstram que o coproduto pode representar uma alternativa viável para a alimentação de ovinos em terminação.

\section{REFERÊNCIAS}

AZEVEDO, R.A.; RUFINO, L.M.A.; SANTOS A.C.R. et al. Desempenho de cordeiros alimentados com inclusão de torta de macaúba na dieta. Pesq. Agropec. Bras., v.47, p.16631668, 2012.

BURGER, P.J.; PEREIRA, J.C.; QUEIROZ, A.C. et al. Comportamento ingestivo em bezerros holandeses alimentados com dietas contendo diferentes níveis de concentrado. Rev. Bras. Zootec., v.29, p.236-242, 2000.

CARDOSO, A.R.; CARVALHO, S.; GALVANI, D.B. et al. Comportamento Ingestivo de cordeiros alimentados com dietas contendo diferentes níveis de fibra em detergente neutro. Ciên. Rural, v.36, p.604-609, 2006.

CARVALHO, S. Desempenho e comportamento ingestivo de cabras em lactação alimentadas com dietas contendo diferentes níveis de fibra. 2002. 118f. Dissertação (Doutorado em Zootecnia) - Curso de Pós-graduação em Zootecnia, Universidade Federal de Viçosa, Viçosa, MG.

CARVALHO, S.; PIRES, A.J.V.; SILVA, R.R. et al. Comportamento ingestivo de ovinos Santa Inês alimentados com dietas contendo farelo de cacau. Rev. Bras. Zootec., v.37, p.660-665, 2008.

CARVALHO, S.; RODRIGUES, M.T.; BRANCO, R.H. et al. Comportamento ingestivo de cabras Alpinas em lactação alimentadas com dietas contendo diferentes níveis de fibra em detergente neutro proveniente da forragem. Rev. Bras. Zootec., v.35, p.562-568, 2006.

CHURCH, D.C. (Ed). The ruminant animal: digestive physiology and nutrition. Englewood Cliffs: Reston, 1988. p.108-116.

DADO, R.G.; ALLEN, M.S. Intake limitations, feeding behavior, and rumen function of cows challenged with rumen fill from dietary fiber or inert bulk. J. Dairy Sci., v.78, p.118-133, 1995. 
DULPHY, J.P.; REMOND, B.; THERIEZ, M. Ingestive behavior and related activities in ruminants. In: RUCKEBUSH, Y.; THIVEND, P. (Eds.). Digestive physiology and metabolism in ruminants. Lancaster: MTP, 1980. p.103-122.

FONTENELE, R.M.; PEREIRA, E.S.; CARNEIRO, M.S.S. et al. Consumo de nutrientes e comportamento ingestivo de cordeiros da raça Santa Inês alimentados com rações com diferentes níveis de energia Metabolizável. Rev. Bras. Zootec., v.40, p.12801286, 2011.

GONÇALVES, A.L.; LANA, R.P.; RODRIGUES, M.T. et al. Padrão nictemeral do $\mathrm{pH}$ ruminal $\mathrm{e}$ comportamento alimentar de cabras leiteiras alimentadas com dietas contendo diferentes relações volumoso:concentrado. Rev. Bras. Zootec., v.3, p.1886-1892, 2001.

JOHNSON, T.R.; COMBS, D.K. Effects of prepartum diet, inert rumen bulk, and dietary polyethylene glycol, on dry matter intake of lactating dairy cows. J. Dairy Sci., v.74, p.933944, 1991

MACEDO, C.A.B.; MIZUBUTI, I.Y.; MOREIRA, F.B. et al. Comportamento ingestivo de ovinos recebendo dietas com diferentes níveis de bagaço de laranja em substituição à silagem de sorgo na ração. Rev. Bras. Zootec., v.36, p.1910-1916, 2007.

MENDONÇA, S.S.; CAMPOS, J.M.S.; VALADARES FILHO, S.C. et al. Comportamento ingestivo de vacas leiteiras alimentadas com dietas à base de cana-de-açúcar ou silagem de milho. Rev. Bras. Zootec., v.33, p.723-728, 2004.
MERTENS, D.R. Creating a system for meeting the fiber requirements of dairy cows. J. Dairy Sci., v.80, p.1463-1481, 1997.

NATIONAL research council - NRC. Subcommittee on sheep nutrition. Nutrient requirements of sheep. 6.ed. Washington, D.C.: National Academy Press, 1985. 99p.

PIRES, A.J.V.; VIEIRA, V.F.; SILVA, F.F. et al. Níveis de farelo de cacau (Theobroma cacao) na alimentação de bovinos. Rev. Electron. Vet., v.6, p.1-10, 2005.

POLLI, V.A.; RESTLE, J.; SENNA, D.B. et al. Aspectos relativos à ruminação de bovinos $\mathrm{e}$ bubalinos em regime de confinamento. Rev. Bras. Zootec., v.25, p.987-993, 1996.

SILVA, H.G.O.; PIRES, A.J.V.; SILVA, F.F. et al. Farelo de cacau (Theobroma cocoa L.) e torta de dendê (Elaeis guineensis, Jacq) na alimentação de cabras em lactação: consumo e produção de leite. Rev. Bras. Zootec., v.34, p.1790-1798, 2005.

SILVA, D.J., QUIROZ, A.C. Análise de alimentos (métodos químicos e biológicos). Viçosa: Universidade Federal de Viçosa, 2002. 235p.

SISTEMA para análise estatística e genética SAEG, Viçosa, MG: Fundação Arthur Bernardes, 2007.

VAN SOEST, P.J. Nutritional ecology of the ruminant. 2.ed. Ithaca: Cornell, 1994. 476p. 